\title{
Prevalence and Progression of Stage 0 Macular Hole in Fellow Eyes of Patients with Idiopathic Full-thickness Macular Hole
}

\author{
Ji Woong Chun ${ }^{1}$, Chang Hwan Kim², Ju Young Kim², Hyun Sub $\mathrm{Oh}^{2}$, Soon Hyun $\mathrm{Kim}^{2}$, Oh Woong Kwon², \\ Yong Sung You ${ }^{2}$ \\ ${ }^{1}$ Barun Eye Clinic, Gwang ju, Korea \\ ${ }^{2}$ Nune Eye Hospital, Seoul, Korea
}

Purpose: To assess the prevalence and progression of a stage 0 macular hole in the fellow eye of patients with an idiopathic full-thickness macular hole.

Methods: The fellow eyes of 189 patients who underwent idiopathic full-thickness macular hole surgery were examined by biomicroscopy and spectral domain-optical coherence tomography (SD-OCT). A subset of 21 fellow eyes with a stage 0 macular hole was observed. Changes in the macular hole were evaluated by biomicroscopy and SD-OCT for an average of 29 months.

Results: Among the 21 eyes, 15 showed no change in perifoveal vitreous detachment (71.4\%). Two eyes (9.5\%) developed complete vitreofoveal separation, and one of the two developed a separation after progression to stage 1A. Among 21 eyes, $5(23.8 \%)$ developed above stage $1 \mathrm{~A}$, and one of the five progressed to stage $1 \mathrm{~B}$ after five years, which was successfully treated with vitrectomy and gas tamponade.

Conclusions: Perifoveal vitreous detachment in the fellow eye on SD-OCT, defined as a stage 0 macular hole, occurred at an earlier phase than stage $1 \mathrm{~A}$ macular holes and may progress to an advanced stage. Therefore, patients who undergo macular hole surgery and have a stage 0 macular hole or perifoveal vitreous detachment in the fellow eye should be followed closely.

Key Words: Retinal perforations, Optical coherence tomography, Vitreous detachment

Fellow eyes of those with a macular hole are likely to develop a full-thickness macular hole or have stronger vitreofoveal adhesion than that in normal eyes [1]. The data

Received: June 15, 2020 Final revision: February 12, 2021

Accepted: February 22, 2021

Corresponding Author: Yong Sung You, MD, PhD. Nune Eye Hospital, 408 Teheran-ro, Gangnam-gu, Seoul 06192, Korea. Tel: 82-2-1661-1175, Fax: 82-2-2086-7710, E-mail: yongsung.you@gmail.com

This paper has been presented in 116th annual meeting of the Korean ophthalmological Society in Korea, November 4, 2016. on the incidence of bilateral macular holes vary considerably from around $5 \%$ to $16 \%$. However, no macular holes were reported to occur in the fellow eye when the vitreous was detached [2]. Persistent vitreofoveal adhesion in eyes with perifoveal vitreous detachment (PVD) is thought to be the leading cause of macular holes [3,4]. In 2004, Chan et al. [5] proposed the concept of stage 0 macular hole to designate fellow eyes with a normal macular figure on optical coherence tomography (OCT) but a posterior hyaloid still attached to the foveal center. They proposed calling cases with partial PVD but no foveal cyst "stage 0 macular 
hole," and estimated an almost six-fold increase in risk for patients with stage 0 macular holes in their fellow eyes. However, a study by Chan et al. [5]. was limited by the use of OCT1, which did not allow for a sufficiently accurate assessment of vitreofoveal adhesion.

Today, the use of spectral domain-OCT (SD-OCT) shows slight changes in the foveolar structure before the occurrence of the early foveal cyst that characterizes a stage 1 macular hole or an impending hole. Characteristically, it appears as a slight focal elevation of the inner curvature of the foveal center, minor changes in the cone outer segment tips (COST), a line at the foveal center, and subtle changes in the reflectivity of the center of the foveola along an anteroposterior axis extending from the internal limiting membrane to the inner segment/outer segment junction line. We anticipate that the early diagnosis of and intervention for vitreomacular changes such as a stage 0 macular hole in the fellow eye of one with a macular hole could be useful in preventing a full-thickness macular hole. To our knowledge, there are no reports on the incidence and prognosis of stage 0 macular holes. The purpose of the current study was to assess the prevalence and progression of stage 0 macular holes in the fellow eyes of patients who underwent idiopathic full-thickness macular hole surgery.

\section{Materials and Methods}

This was an observational, retrospective case series using Spectralis SD-OCT (Heidelberg Engineering, Heidelberg, Germany) images to examine the vitreoretinal interface and foveal morphologic features in stage 0 macular holes. We reviewed the charts of 189 asymptomatic fellow eyes of patients who underwent idiopathic full-thickness macular hole surgery at Nune Eye Hospital from January 2010 through June 2016. We excluded patients with high myopia ( $>6$ diopters) or with a history of ocular trauma, and cases in which the fellow eyes had undergone retinal photocoagulation or surgery for complications of retinochoroidal diseases. We used the criteria of the grading system established by Gass [6]. Stage 1 macular hole, or impending macular hole, was demonstrated by a loss of the foveal depression. Stage 1A was a foveolar detachment characterized by the loss of the foveal contour and a lipofuscin-colored spot. Stage 1B was a foveal detachment

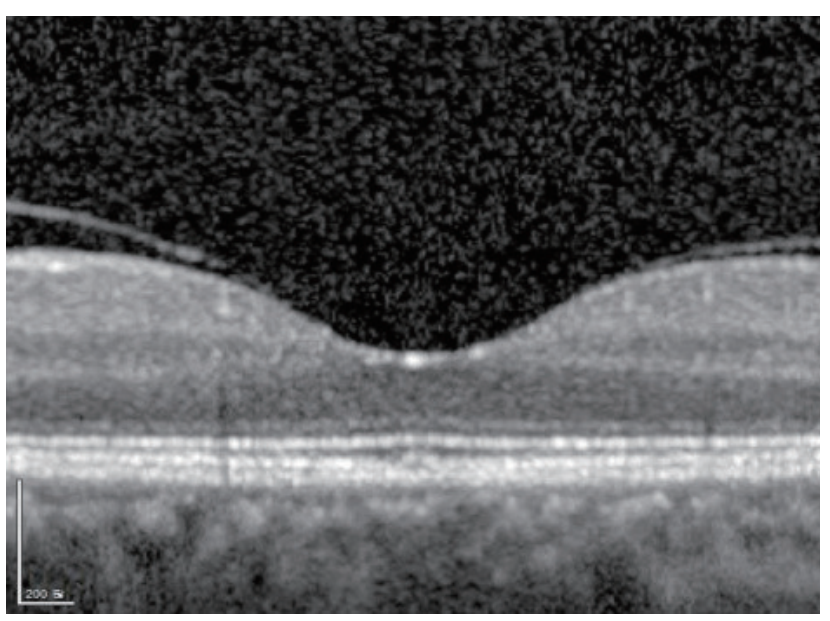

Fig. 1. Perifoveal vitreous detachnment was defined as a detached posterior hyaloid but attached only to the fovea with a complete perifoveal detachment on spectral domain-optical coherence tomography.

characterized by a lipofuscin-colored ring. Stage 2 macular hole was defined by a full-thickness break $<400 \mu \mathrm{m}$ in size. Stage 3 macular hole was defined as a further progression to a hole $\geq 400 \mu \mathrm{m}$ in size. The posterior hyaloid is noted to be detached over the macula with or without an overlying operculum. Stage 4 macular hole was characterized by a stage 3 macular hole with a complete posterior vitreous detachment and Weiss ring.

The study was approved by the institutional review board of Nune Eye Hospital and adhered to the tenets of the Declaration of Helsinki. Informed consent was waived because of the retrospective nature of the study. We classified the features of the vitreoretinal interface by bio-microscopy or using serial SD-OCT images, and defined PVD as a detached posterior hyaloid, attached only to the fovea with complete perifoveal detachment on SD-OCT (Fig. 1). All patients were examined by SD-OCT imaging through dilated pupils. Six-millimeter line scans were performed through the central macula along with a 2-dimensional raster scan and a 3 -dimensional reconstruction of the macula centered over the fovea.

\section{Results}

Among 189 patients who underwent full-thickness macular hole surgery, 21 fellow eyes were found to have a stage 0 macular hole. We included a total of 21 fellow eyes 
Table 1. Clinical characteristics of patients with stage 0 macular holes

\begin{tabular}{llc}
\hline Characteristics & & Value \\
\hline Age (yr) & & $63.1 \pm 9.58$ \\
Male : female & Stage 1B & $7(33.3): 14(66.7)$ \\
Macular hole stage of affected eye & Stage 2 & 3 Eyes $(14.2)$ \\
& Stage 3 & 13 Eyes $(61.9)$ \\
& Stage 4 & 4 Eyes (19.0) \\
& Baseline & 1 Eye (4.9) \\
Mean horizontal diameter ${ }^{*}(\mu \mathrm{m})$ & Last follow-up & $1,008.6$ \\
& & 866 \\
Follow-up period (mon) & & $28.45 \pm 23.5$ \\
Best-corrected visual acuity (logMAR) & & $0.37 \pm 0.23$ \\
Spherical equivalent refraction (diopter) & & $-0.34 \pm 1.73$ \\
Lens status (phakic : pseudophakic) & & $18(85.7): 3(14.3)$ \\
Axial length (mm) & & $24.49 \pm 0.99$ \\
\hline
\end{tabular}

Values are presented as mean \pm standard deviation or number (\%). $\log \mathrm{MAR}=\operatorname{logarithm}$ of the minimum angle of resolution.

*Attachment area of the vitreous at the fovea.

Stage $0 \mathrm{MH}(\mathrm{n}=21)$

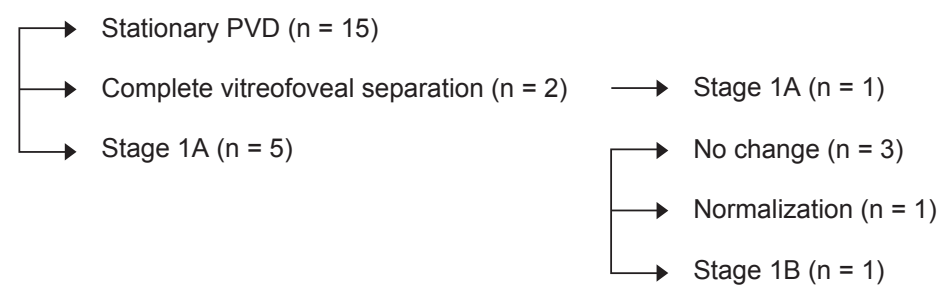

\begin{tabular}{|c|c|c|c|c|c|c|c|c|}
\hline Case no. & Sex & $\begin{array}{l}\text { Age } \\
(\mathrm{yr})\end{array}$ & $\begin{array}{c}\text { BCVA } \\
\text { (logMAR) }\end{array}$ & $\begin{array}{l}\text { Lens } \\
\text { status }\end{array}$ & $\begin{array}{l}\text { MH stage of the } \\
\text { affected eye at } \\
\text { baseline }\end{array}$ & $\begin{array}{l}\text { Horizontal diameter } \\
\text { of attachment area } \\
(\mu \mathrm{m})\end{array}$ & Change in $\mathrm{MH}$ stage & $\begin{array}{c}\text { Time of MH } \\
\text { stage change } \\
\text { (mon) }\end{array}$ \\
\hline 1 & Female & 63 & 0.39 & Phakic & Stage 3 & 1,288 & $1 \mathrm{~A} \rightarrow 1 \mathrm{~B}$ & 62 \\
\hline 2 & Female & 58 & 0.04 & Phakic & Stage 2 & 1,404 & $\begin{array}{l}\text { Complete vitreofoveal } \\
\text { separation } \rightarrow 1 \mathrm{~A}\end{array}$ & 32 \\
\hline 3 & Female & 68 & 0 & Phakic & Stage 4 & 774 & $1 \mathrm{~A} \rightarrow$ normalization & 30 \\
\hline 4 & Female & 67 & 0 & Phakic & Stage 2 & 986 & $1 \mathrm{~A} \rightarrow 1 \mathrm{~A}$ & 48 \\
\hline 5 & Male & 68 & 0 & Phakic & Stage 1B & 1,098 & $1 \mathrm{~A} \rightarrow 1 \mathrm{~A}$ & 31 \\
\hline 6 & Male & 46 & 0 & Phakic & Stage 2 & 1,308 & $1 \mathrm{~A} \rightarrow 1 \mathrm{~A}$ & 6 \\
\hline
\end{tabular}

Fig. 2. Changing stage 0 macular holes in the fellow eye during the follow-up period. $M H=$ macular hole; $P V D=$ perifoveal vitreous detachment; $\mathrm{BCVA}=$ best-corrected visual acuity; $\log \mathrm{MAR}=\operatorname{logarithm}$ of the minimum angle of resolution.

of 189 patients in this study. The clinical characteristics of the 21 patients with stage 0 macular holes are shown in Table 1 . The mean age of 21 patients was $63.1 \pm 9.58$ years with a range of 44 to 77 years. Seven patients were male $(33.3 \%)$ and 14 were female (66.7\%). Three of the 21 fellow eyes $(14.2 \%)$ had a stage 1B macular hole, $13(61.9 \%)$ had a stage 2 hole, four (19.0\%) had a stage 3 hole, and one
(4.9\%) had a stage 4 hole. The mean follow-up period was $28.45 \pm 23.5$ months with a range of 6 to 73 months. During the follow-up periods, 15 eyes of the $21(71.4 \%)$ showed stationary PVD, and two (9.5\%) showed complete vitreofoveal separation. Of these two eyes, one had complete vitreofoveal separation after the progression to a stage 1A macular hole. Five of the 21 eyes (23.8\%) pro- 

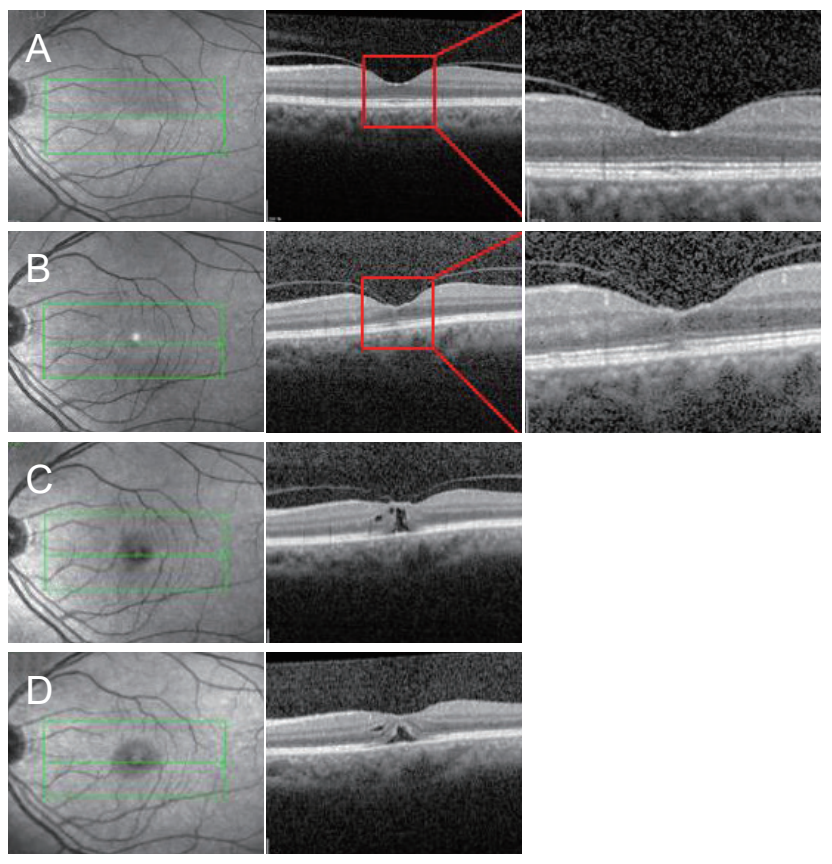

Fig. 3. (A) Horizontal optical coherence tomography scan of fellow eye of a macular hole showing the posterior hyaloid slightly detached from the macular surface, except at the edge of the foveal pit. (B) After 5 years, a change in the inner foveal curvature at the point of traction exerted by the posterior hyaloid. A small section of the cone outer segment tips line and inner segment/ outer segment line and external limiting membrane are detached. (C) Two months later, the posterior hyaloid is still attached to the roof of the cyst. There is a break in the photoreceptor layer. (D) After postoperative 2 months, the posterior hyaloid is removed by vitrectomy. The cystic cavity and central defect are shrank. The green line represents the macular scan area, and the red line represents the magnification area.

gressed to stage 1A macular holes, and of those five, one progressed to stage $1 \mathrm{~B}$ after 5 years; this eye underwent a vitrectomy and gas tamponade (Fig 2). The mean follow-up period was $28.41 \pm 15.96$ months, with a range of 9 to 58 months. During the follow-up periods, 5 of 12 eyes underwent vitrectomy, and the mean duration until surgery was $17.8 \pm 23.02$ months, with a range of 2 to 57 months. As a representative case, a 63-year-old woman was referred for surgery for a stage 3 full-thickness macular hole in her right eye. In the left eye, the best-corrected visual acuity was 1.0 , no posterior vitreous detachment was found, and the macula appeared normal on biomicroscopy. Horizontal SD-OCT revealed a normal macular appearance and a posterior hyaloid slightly detached from the macular surface except at the edge of the foveal pit. Five years later, her best-corrected visual acuity had decreased to 0.6 in the left eye, and the horizontal SD-OCT revealed a change in the inner foveal curvature at the point of traction exerted by the posterior hyaloids. A small section of the COST line and the inner segment/outer segment line and external limiting membrane were detached. Two months later, her best-corrected visual acuity had decreased to 0.3 in the left eye, and the horizontal SD-OCT revealed that the posterior hyaloid was still attached to the roof of the cyst but there was a break in the photoreceptor layer, indicating a stage 1B macular hole. This patient subsequently underwent successful pars plana vitrectomy and gas tamponade of the left eye. Two months after surgery, the best-corrected visual acuity was improved to 0.4 . Postoperative horizontal SD-OCT revealed that the posterior hyaloids were removed by vitrectomy and the cystic cavity and central defect of the fovea improved (Fig. 3A-3D).

\section{Discussion}

It is widely believed that patients with the earliest stages of macular holes have localized perifoveal PVD with a persistent vitreous attachment to the fovea. This attachment at the fovea is thought to be a mechanism by which vitreous traction could exert its effects on the fovea, leading to the development of a full-thickness macular hole. Therefore, vitreofoveal attachment of a perifoveal PVD in the fellow eye on SD-OCT in patients with a unilateral idiopathic macular hole is a significant risk factor for the development of a second macular hole [5,7]. However, it remains unclear which eyes will proceed to spontaneous resolution or exhibit a full-thickness macular hole.

We investigated the prevalence and prognosis of stage 0 macular holes in the fellow eye of patients who underwent idiopathic macular hole surgery. We selected fellow eyes with a stage 0 macular hole because eyes that progress to idiopathic full-thickness macular holes or become free from the risk of an idiopathic full-thickness macular hole must be this stage before progressing to the next stage.

Chan et al. [5] introduced the concept of a stage 0 macular hole based on OCT observations of the vitreoretinal interface in the fellow eyes of patients with unilateral idiopathic macular holes and to evaluate the subsequent risk of progression to a full-thickness macular hole. In their retrospective study of 94 fellow eyes, macular holes only occurred in $4.3 \%$ of the cases without visible vitreofoveal adhesion and in $42 \%$ of the cases with a stage 0 macular hole. 
Majji et al. [8] observed that $30 \%$ of the patients had stage 0 macular hole changes in the fellow eye. Chan et al. [5] reported that $28.7 \%$ of the patients had a stage 0 macular hole. Uemura et al. [9] reported that among 55 normal fellow eyes, 11 eyes $(20 \%)$ had a triangular elevation of the COST line at the center of the fovea on SD-OCT, corresponding to a stage 0 macular hole. In our study, we observed that 21 eyes (11.1\%) of 189 patients had stage 0 macular hole changes in the fellow eye. The prevalence of stage 0 macular holes was somewhat lower than that in previous studies.

The prevalence of developing a full-thickness macular hole in the fellow eye of patients with a unilateral full-thickness macular hole was reported to vary widely. Niwa et al. [7] noted that this may be due to difficulty in evaluating the vitreous. Uemura et al. [9] reported that 2 (11.1\%) of the 18 fellow eyes with unilateral idiopathic macular holes developed full-thickness macular holes. Majji et al. [8] reported that 11 (17.1\%) of 64 fellow eyes with unilateral idiopathic full-thickness macular holes had stage 3, 4 full-thickness macular holes. Niwa et al. [7] also reported that 3 (5.1\%) of 58 fellow eyes with unilateral idiopathic macular holes developed stage 2 full-thickness macular holes. However, the studies did not accurately identify the macular hole stages. In our study, we found that five (23.8\%) of the 21 eyes progressed to stage $1 \mathrm{~A}$ macular holes. One (4.8\%) of the 21 eyes developed a stage 1B macular hole during the follow-up period and pars plana vitrectomy and gas tamponade were performed. If there was no early intervention for a stage $1 \mathrm{~B}$ macular hole after close follow-up, the possibility of becoming a full-thickness macular hole was very high.

Although a comparison to previous studies was difficult because of different stages of full-thickness macular holes and variable follow-up periods, we anticipate that the early diagnosis of and intervention for vitreomacular changes such as stage 0 macular holes in the fellow eyes of patients with full-thickness macular holes could be useful in preventing the progression to full-thickness macular holes.

There were several limitations to this study because it was not a prospective study. The number of patients was small and the follow-up periods varied. In addition, the evaluations of the SD-OCT findings were subjective.

In conclusion, a stage 0 macular hole is considered to be important as one of the first changes observed in macular tissue in full-thickness macular hole formation. Patients with stage 0 macular holes on SD-OCT in the fellow eye must be followed closely as progression to an advanced stage may result in decreased visual acuity, requiring early intervention. Further studies with prospective and larger sample sizes are needed to understand the nature of stage 0 macular holes.

\section{Conflict of Interest}

No potential conflict of interest relevant to this article was reported.

\section{References}

1. Akiba J, Kakehashi A, Arzabe CW, Trempe CL. Fellow eyes in idiopathic macular hole cases. Ophthalmic Surg 1992;23:594-7.

2. Kumagai K, Hangai M, Larson E, Ogino N. Vitreoretinal interface and foveal deformation in asymptomatic fellow eyes of patients with unilateral macular holes. Ophthalmology 2011;118:1638-44.

3. Chauhan DS, Antcliff RJ, Rai PA, et al. Papillofoveal traction in macular hole formation: the role of optical coherence tomography. Arch Ophthalmol 2000;118:32-8.

4. Haouchine B, Massin P, Gaudric A. Foveal pseudocyst as the first step in macular hole formation: a prospective study by optical coherence tomography. Ophthalmology 2001;108:15-22

5. Chan A, Duker JS, Schuman JS, Fujimoto JG. Stage 0 macular holes: observations by optical coherence tomography. Ophthalmology 2004;111:2027-32.

6. Gass JD. Idiopathic senile macular hole: its early stages and pathogenesis. Arch Ophthalmol 1988;106:629-39.

7. Niwa H, Terasaki H, Ito Y, Miyake Y. Macular hole development in fellow eyes of patients with unilateral macular hole. Am J Ophthalmol 2005;140:370-5.

8. Majji AB, Chhablani JK, Bagga B. Vitreo-retinal interface changes on optical coherence tomography in the fellow eyes of patients with macular hole. Int $J$ Ophthalmol 2013;6:526-30.

9. Uemura A, Otsuji F, Nakano T, Sakamoto T. Vitreomacular interface and outer foveal microstructure in fellow eyes of patients with unilateral macular holes. Retina 2014;34:1229-34. 